\title{
Good correlation between the Afinion AS100 analyser and the ABX Pentra 400 analyser for the measurement of glycosylated haemoglobin and lipid levels in older adults in Durban, South Africa
}

\author{
N S Abbai, ${ }^{1} \mathrm{PhD} ; \mathrm{M}$ Nyirenda, ${ }^{2} \mathrm{PhD} ;$ T Reddy, ${ }^{3} \mathrm{PhD}$; G Ramjee, ${ }^{2,4,5} \mathrm{PhD}$; on behalf of the SHIOP team \\ ${ }^{1}$ School of Clinical Medicine Research Laboratory, Nelson Mandela School of Medicine, University of KwaZulu-Natal, Durban, South Africa \\ ${ }^{2}$ HIV Prevention Research Unit, South African Medical Research Council, Durban, South Africa \\ ${ }^{3}$ Biostatistics Unit, South African Medical Research Council, Durban, South Africa \\ ${ }^{4}$ Department of Epidemiology and Population Health, London School of Hygiene and Tropical Medicine, UK \\ ${ }^{5}$ Department of Global Health, School of Medicine, University of Washington, USA
}

Corresponding author: NS Abbai (abbain@ukzn.ac.za)

Background. The Afinion AS100 analyser is a small bench-top, multi-assay, point-of-care (POC) analyser that is able to measure glycated haemoglobin (HbAlc) and lipid levels.

Objective. To assess performance of the Afinion analyser compared with a reference laboratory test for the measurement of HbAlc and lipid levels.

Method. The study involved men and women enrolled in a cross-sectional study, Sexual health, HIV infection and comorbidity with non-communicable diseases among Older Persons (SHIOP), which was conducted from February to May 2016. Whole blood was drawn aseptically by a trained study nurse into a serum separator gel tube and an ethylenediaminetetra-acetic acid (EDTA) tube. The EDTA whole blood was used to measure HbAlc levels, and serum to measure total cholesterol (TC), high-density lipoprotein cholesterol (HDL-C), lowdensity lipoprotein cholesterol (LDL-C) and triglyceride levels. Lin's correlation coefficient was used to assess the agreement between the Afinion and ABX Pentra 400 analysers for each marker.

Results. A total of 435 older individuals were included in the study. The proportion of HbA1c results that were correctly classified by the Afinion analyser was $92.2 \%$. Bland-Altman analysis and linear regression analysis showed a very good agreement (correlation concordance 0.89 ) between the two analysers for the measurement of HbAlc. The two-way scatter plot for TC showed a substantial correlation $(0.80)$. However, a total of 69 cholesterol results that were within the normal range on the Pentra were misclassified as abnormal on the Afinion. The readings obtained for HDL-C levels with the Afinion were shown to be slightly overestimated when compared with the Pentra. However, correlation for HDL-C on the two analysers was 0.93 , indicating an almost perfect agreement. Seventy-four LDL-C results were erroneously classified as abnormal on the Afinion but were within the normal range on the Pentra, resulting in a substantial correlation of 0.75 . An excellent agreement was observed between triglyceride measurements (0.99).

Conclusion. This study supports the use of the Afinion AS100 analyser in POC testing for the measurement of HbAlc, triglycerides and HDL-C in a South African setting.

S Afr Med J 2018;108(1):50-55. DOI:10.7196/SAMJ.2018.v108i1.12548

Globally, 18 million people die annually from cardiovascular disease (CVD).$^{[1]}$ Findings from the INTERHEART Africa study ${ }^{[2]}$ indicated that the highest number of premature acute myocardial infarctions in the world occur in sub-Saharan Africa, as a result of lack of early detection of CVD and effective management of risk factors. ${ }^{[3]}$ Various point-of-care (POC) tests (mainly finger-prick tests) are available that provide results ranging from total cholesterol (TC) alone to a full lipogram. However, the limitation with many of these tests is that the results obtained are not adequate to commit a patient to a lifetime of therapy. In addition, finger-prick testing that measures TC alone will not detect raised triglycerides. ${ }^{[3]}$

Patients with diabetes mellitus (DM) are at high risk of developing CVD, with increased associated mortality. ${ }^{[4]}$ In many primary care settings, testing for glycaemic control involves sending a blood sample away for laboratory testing and waiting a number of days for the result to be returned. This delays patient counselling and treatment adjustments based on glycated haemoglobin (HbAlc) levels, and sometimes follow-up can be lost completely. ${ }^{[4]}$ Measurement of $\mathrm{HbAlc}$ levels using POC tests provides rapid results, improving patient management. ${ }^{[4-6]}$ Findings from a randomised controlled trial of POC testing in an Australian setting showed that POC tests of samples from patients with established hyperlipidaemia or established type 1 or type $2 \mathrm{DM}$, or taking anticoagulant therapy, had the same clinical effectiveness as testing in a pathology laboratory. ${ }^{[7]}$ Reports have also shown that access to a POC test is associated with improved treatment adherence. ${ }^{[8]}$

The Afinion AS100 analyser (Alere, South Africa (SA)) is a small bench-top, multi-assay analyser for in vitro diagnostic POC testing using whole blood, plasma or urine samples. The unique feature of the analyser is that both the HbAlc and the lipid panel test can be done on one instrument. The turnaround time from collection of samples to all results being available to the patient is $<30$ minutes. 
Preliminary results from a recent study on sexual health and chronic morbidities in people aged $\geq 50$ years conducted in the Chatsworth and Botha's Hill areas of Durban, SA, found that $>70 \%$ of older adults had elevated blood glucose levels indicating prediabetes and diabetes, and that over half of the study participants had elevated blood pressure readings (unpublished data). In addition, $>15 \%$ of the study participants were HIV-infected, which is higher than the national average. ${ }^{[9]}$ There is therefore an urgent need to respond to the growing twin health challenges of HIV and non-communicable diseases among older adults.

\section{Objective}

To evaluate the performance of the lipid and $\mathrm{HbAlc}$ panels of the Afinion AS100 analyser compared with standard laboratory methods in a population of HIV-positive and HIV-negative older adults.

\section{Methods \\ Study participants}

The study was a sub-analysis of a cross-sectional study, Sexual health, HIV infection and comorbidity with non-communicable diseases among Older Persons (SHIOP). The SHIOP study enrolled both men and women and was conducted between February and May 2016. The primary aim of SHIOP was to describe sexuality, sexual health and the comorbidity of HIV and sexually transmitted infections with chronic non-communicable diseases in adults aged $\geq 50$ years in a setting of high HIV prevalence. Participants were recruited from two sub-areas of Durban (Botha's Hill and Chatsworth), SA. Botha's Hill is a semi-rural area located west of Durban, and Chatsworth is an urban setting located south of Durban. The study eligibility criteria included age $\geq 50$ years, being willing and able to provide written informed consent and able to communicate in English or isiZulu, and not being terminally ill or cognitively impaired.

\section{Ethical considerations}

As part of the study procedure, all study participants provided written informed consent prior to enrolment. Participants who were illiterate (i.e. unable to read or write English or isiZulu), were assisted by an impartial literate witness during the informed consent process. The participant used a thumbprint to mark the informed consent form and the impartial witness signed to confirm that the participant provided informed consent. We followed the usual procedure adopted in large-scale multi-site HIV prevention clinical trials. Ethical approval for SHIOP was obtained from the South African Medical Research Council (SAMRC) Ethics Committee (ref. no. EC030-9/2015).

\section{Sample collection and processing}

All eligible participants were requested to provide a venous blood sample for laboratory testing. Whole blood was drawn aseptically by a trained study nurse into a serum separator gel tube and an ethylenediaminetetra-acetic acid (EDTA) tube. The serum gel tube was centrifuged at the research clinics and the separated serum used for measuring lipid levels, and the EDTA whole-blood tube was used to measure $\mathrm{HbAlc}$ levels using the POC Afinion AS100 analyser, which was placed at the research clinics. The remaining serum and EDTA whole blood was transported on ice on the day of collection to the SAMRC HIV Prevention Research Unit's central routine laboratory for measurement of blood glucose and lipid levels on the ABX Pentra 400 analyser (Horiba, USA). All testing was performed by trained medical technologists.

\section{POC testing using the Afinion analyser}

\section{HbAlc measurements}

HbA1c levels were measured using $1.5 \mu \mathrm{L}$ of EDTA whole blood. The Afinion works on the boronic acid affinity test principle and is not affected by haemoglobin variants. The analyser claims a $4.0-15.0 \%$ (20.0 - $140.0 \mathrm{mmol} / \mathrm{mol}$ ) HbAlc measuring range. The assay time was $\sim 3$ minutes, and controls with HbA1c-specific target values were included in the assay runs.

\section{Lipid measurements}

TC, low-density lipoprotein cholesterol (LDL-C), high-density lipoprotein cholesterol (HDL-C) and triglycerides were measured using $1.5 \mu \mathrm{L}$ of serum. The assay time was $\sim 8$ minutes, and ready-touse control material was included in the assay runs. The measuring ranges for lipids using the Afinion analyser were as follows: TC 2.59 $12.95 \mathrm{mmol} / \mathrm{L}$, HDL-C 0.39 - $2.59 \mathrm{mmol} / \mathrm{L}$, and triglycerides 0.51 $7.34 \mathrm{mmol} / \mathrm{L}$. LDL-C was a computed value using the Friedewald formula ${ }^{[10,11]} \mathrm{LDL}-\mathrm{C}(\mathrm{mmol} / \mathrm{L})=\mathrm{TC}-\mathrm{HDL}-\mathrm{C}-$ triglycerides/2.2.

\section{Reference laboratory testing}

The Pentra analyser was used as the reference laboratory test according to the manufacturer's instructions. According to SA national guidelines, the reference (normal) range for testing and management of lipids is as follows: TC $<4.5 \mathrm{mmol} / \mathrm{L}$, LDL-C $<2.5 \mathrm{mmol} / \mathrm{L}, \mathrm{HDL}-\mathrm{C} 1.0 \mathrm{mmol} / \mathrm{L}$ (men) and $\geq 1.2 \mathrm{mmol} / \mathrm{L}$ (women), and triglycerides $<1.70 \mathrm{mmol} / \mathrm{L}$. The diagnostic cut-off for $\mathrm{HbAlc}$ is $6.5 \%$ (or $48 \mathrm{mmol} / \mathrm{mol}$ ). We used these reference ranges to compare participants categorised as normal v. abnormal on the Pentra and Afinion for each marker.

\section{Data analysis}

Lin's correlation coefficient was used to assess the agreement between the Afinion and Pentra analysers for each marker. The mean bias was calculated, as well as the $95 \%$ limits of agreement to quantify the difference between a reading on the Afinion and the Pentra. Results were graphically depicted using Bland-Altman plots and two-way scatterplots. The coefficients were classified as indicating poor $(<0.69)$, fair (0.70 - 0.79), good (0.80 - 0.89) or excellent $(0.90$ - 1.00) correlation.

\section{Results \\ Sample description}

We obtained data on 435 older adults from Durban, SA. The overall median age of the study participants was 61 years (interquartile range 12), with male participants being slightly older than female participants (62 v. 60 years). Using World Health Organization categorisations of clinically assessed weight and height, $\sim 60 \%$ of study participants were overweight or obese, and $\sim 40 \%(n=172)$ were hypertensive. Furthermore, $16.1 \%$ of participants $(n=70)$ were HIVpositive, of whom $84.3 \%(n=59)$ knew their HIV status prior to study participation (data not shown).

Diagnostic accuracy of the Afinion v. the Pentra analysers Table 1 describes the diagnostic performance of the Afinion analyser for the measurement of $\mathrm{HbAlc}$, TC, LDL-C, HDL-C and triglyceride levels in comparison with the Pentra. In addition, the differences between the Afinion and Pentra measurements using Bland-Altman plots are described in Table 2 and Figs 1 - 5.

\section{HbAlc levels}

The proportion of $\mathrm{HbAlc}$ results that were correctly classified by the Afinion analyser was $92.2 \%$ (Table 1). We also observed high diagnostic 
Table 1. Diagnostic accuracy of the Afinion analyser compared with the Pentra, using target levels as cutoffs

\begin{tabular}{|c|c|c|c|c|c|c|}
\hline & & \multicolumn{2}{|c|}{ Pentra } & \multicolumn{3}{|c|}{ Afinion } \\
\hline & & Normal, $n$ & Abnormal, $n$ & $\begin{array}{l}\text { Correctly } \\
\text { classified, \% }\end{array}$ & Sensitivity, \% & Specificity, \% \\
\hline \multirow[t]{3}{*}{$\mathrm{HbAlc}$} & Afinion & & & & & \\
\hline & Normal & 214 & 7 & 92.2 & 90.9 & 92.6 \\
\hline & Abnormal & 17 & 70 & & & \\
\hline \multirow[t]{3}{*}{ TC } & Afinion & & & & & \\
\hline & Normal & 122 & 0 & 79.3 & 100 & 63.9 \\
\hline & Abnormal & 69 & 142 & & & \\
\hline \multirow[t]{3}{*}{ LDL-C } & Afinion & & & & & \\
\hline & Normal & 126 & 1 & 76.1 & 99.1 & 63.0 \\
\hline & Abnormal & 74 & 113 & & & \\
\hline \multirow[t]{3}{*}{ HDL-C, males } & Afinion & & & & & \\
\hline & Normal & 12 & 2 & 90.9 & 97.5 & 63.2 \\
\hline & Abnormal & 7 & 78 & & & \\
\hline \multirow[t]{3}{*}{ HDL-C, females } & Afinion & & & & & \\
\hline & Normal & 76 & 5 & 89.9 & 96.3 & 80.9 \\
\hline & Abnormal & 18 & 129 & & & \\
\hline \multirow[t]{3}{*}{ TG } & Afinion & & & & & \\
\hline & Normal & 158 & 15 & 95.4 & 91.1 & 100 \\
\hline & Abnormal & 0 & 154 & & & \\
\hline
\end{tabular}

\section{Table 2. Differences between the Afinion and Pentra measurements using BA plots}

\begin{tabular}{lllll}
\hline & $N$ & Mean bias (SD) & 95\% BA limits of agreement & Concordance correlation \\
\hline HbA1c & 308 & $0.224(0.840)$ & $-1.421-1.870$ & 0.89 \\
TC & 333 & $0.569(0.377)$ & $-0.169-1.307$ & 0.80 \\
LDL-C & 314 & $0.528(0.385)$ & $-0.227-1.283$ & 0.75 \\
HDL-C & 327 & $0.089(0.095)$ & $-0.097-0.275$ & 0.93 \\
TG & 327 & $-0.124(0.127)$ & $-0.373-0.125$ & 0.99 \\
BA = Bland-Altman; SD = standard deviation; HbAlc = glycated haemoglobin; TC = total cholesterol; HDL-C = high-density cholesterol; LDL-C = low-density cholesterol; TG = trigycerides.
\end{tabular}

accuracy of the Afinion in detecting results that were above target levels, as indicated by both sensitivity and specificity $>90 \%$. According to our analysis, higher values for $\mathrm{HbAlc}$ levels were detected with the Afinion than with the Pentra. Bland-Altman analysis and linear regression analysis showed an almost excellent agreement between the two analysers (Fig. 1). A correlation of 0.89 was observed (Table 2).

\section{Lipid levels}

The proportion of TC results that were correctly classified by the Afinion analyser was $79.3 \%$ (Table 1). The TC two-way scatter plot represented in Fig. 2 showed a good correlation $(0.80)$ between the two analysers. However, a total of 69 TC results that were within the normal range on the Pentra were misclassified as abnormal on the Afinion, leading to a poor specificity of $63.9 \%$. However, the Afinion correctly classified all 142 results that were in the abnormal range on the Pentra, resulting

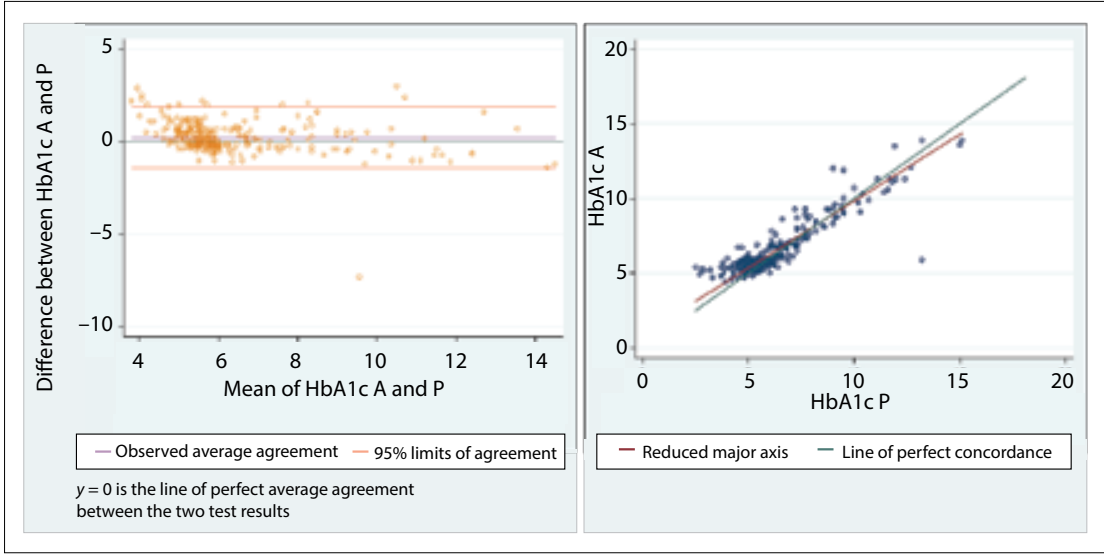

Fig. 1. Scatterplot and regression line of $H b A 1 c$ values (\%) produced by the Afinion (A) and Pentra (P) analysers. The Bland-Altman study and linear regression analysis showed good agreement between the two analysers. $(H b A 1 c=$ glycated haemoglobin. $)$

in a sensitivity of $100 \%$. The mean bias observed for TC measurements between the Afinion and the Pentra analysers was 0.569 , with $95 \%$ limits of agreement of $-0.169-1.307$ (Table 2).
The readings obtained for HDL-C levels with the Afinion were shown to be slightly overestimated compared with the Pentra (mean bias 0.089). The correlation between the two methods was 0.93 , indicating excel- 


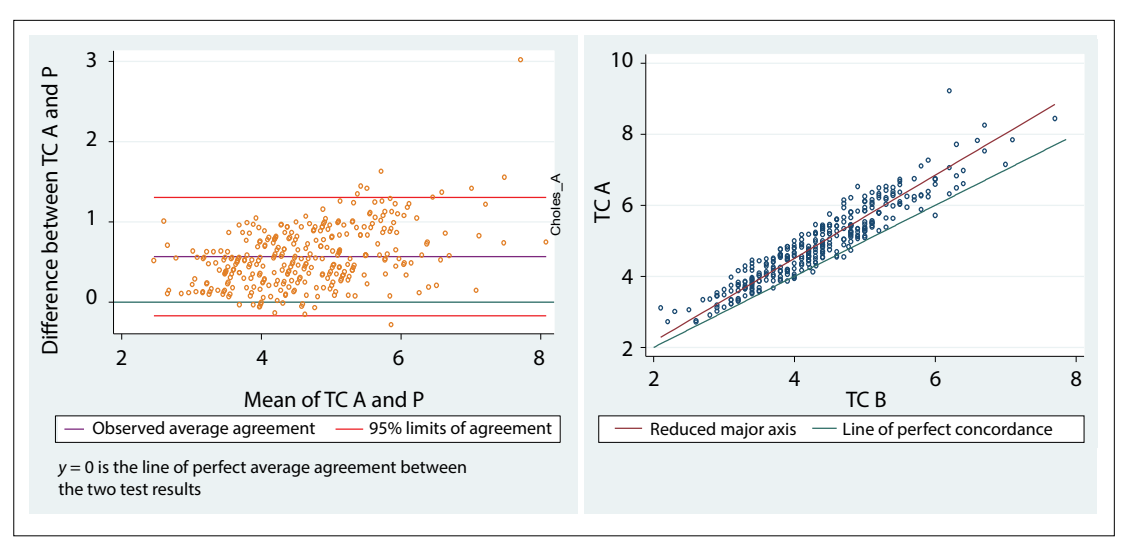

Fig. 2. Scatterplot and regression line of TC values ( $m m o l / L)$ produced by the Afinion (A) and Pentra $(P)$ analysers. The Bland-Altman study and linear regression analysis showed a substantial agreement between the two analysers. $(T C=$ total cholesterol. $)$

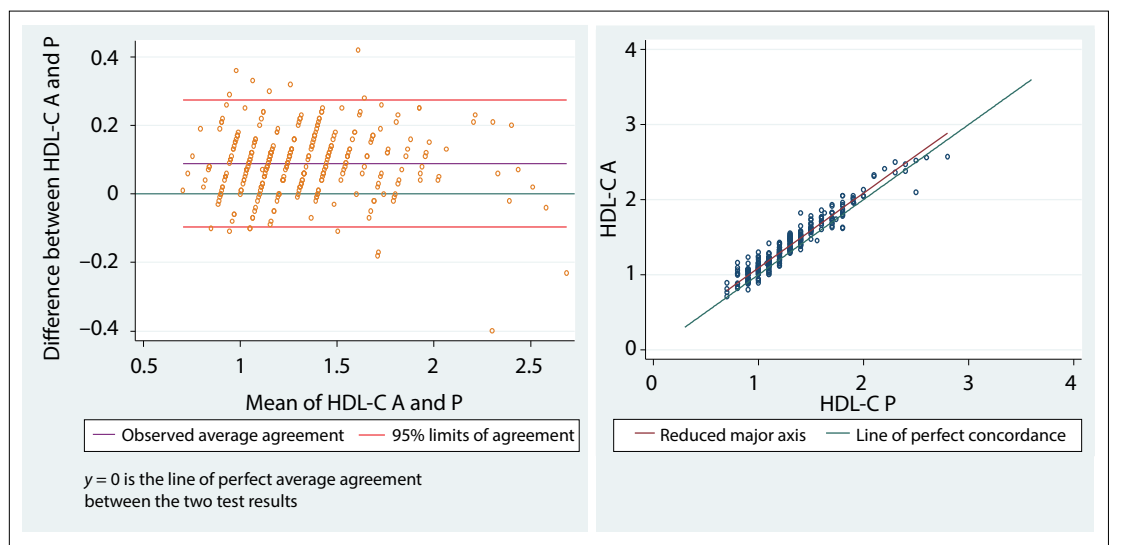

Fig. 3. Scatterplot and regression line of HDL-C values $(\mathrm{mmol} / \mathrm{L})$ produced by the Afinion $(A)$ and Pentra $(P)$ analysers. The Bland-Altman study and linear regression analysis showed an almost perfect agreement between the two analysers. (HDL-C = high-density lipoprotein cholesterol.)

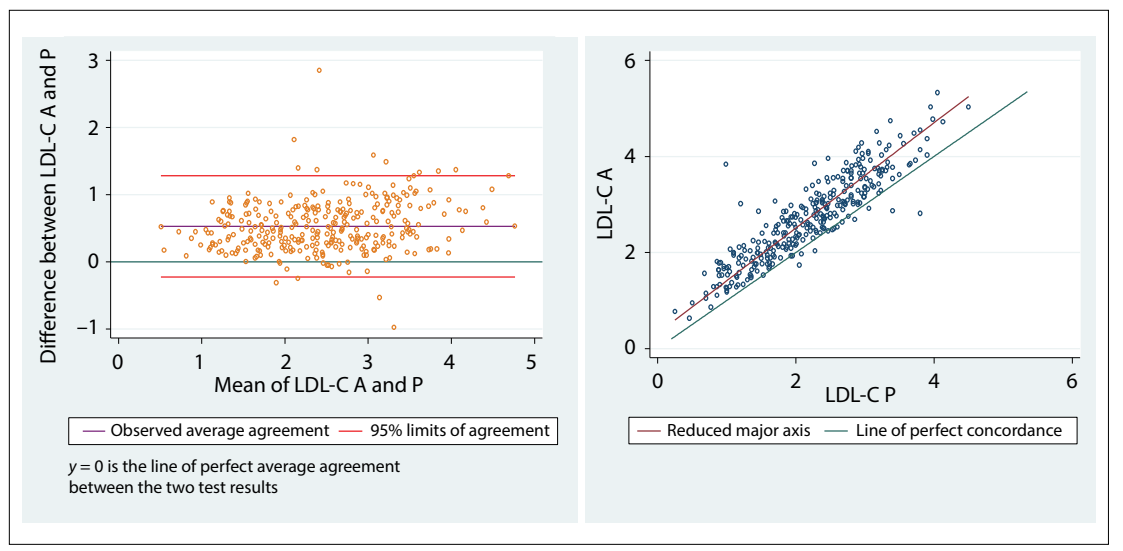

Fig. 4. Scatterplot and regression line of LDL-C values (mmol/L) produced by the Afinion $(A)$ and Pentra $(P)$ analysers. The Bland-Altman study and linear regression analysis showed a substantial agreement between the two analysers. ( $L D L-C=$ low-density lipoprotein cholesterol.)

lent agreement between the two analysers (Fig. 3). Misclassification of HDL-C results was low in both males and females. The specificity of the Afinion test was lower in males, 7 of 19 HDL results that were below the target levels on the Pentra being incorrectly classified as normal by the Afinion. poor specificity was observed, the sensitivity of the Afinion was $99.1 \%$, indicating that the analyser performs well in detecting values above the target range. However, it is important to note that the LDL-C was a computed value using the Friedewald formula, and because it was not measured directly it may not be a direct reflection of the accuracy of the analysers used. Despite this, a fair correlation of 0.75 was observed between the two analysers (Fig. 4).

Excellent agreement was observed between triglycerides measured on the Afinion and the Pentra, evidenced by the mean bias of 0.124 and almost perfect agreement between the two analysers, with a correlation of 0.99 (Fig. 5). When categorising values according to target levels, excellent diagnostic accuracy was observed, with a sensitivity of $91.1 \%$ and specificity of $100 \%$.

\section{Discussion}

The objective of this study was to evaluate the performance of the Afinion AS100 analyser compared with a standard laboratory method for the measurements of lipids and HbAlc in a population of HIVpositive and HIV-negative older adults. Overall, Bland-Altman plots revealed a good correlation between the Afinion and Pentra 400 analysers for the measurement of $\mathrm{HbAlc}$ levels. In addition, the Afinion was able to correctly classify $>90 \%$ of the samples. With regard to the measurement of lipid levels, the results showed very good correlation between the Afinion and the Pentra, with almost perfect correlations noted for HDL-C and triglycerides.

HbA1c measurement is an important tool in the management of patients with DM. Evidence for the utility of HbAlc for the diagnosis and detection of DM in an SA population has been described by Hird et al. ${ }^{[12]}$ However, in SA primary healthcare facilities, random blood glucose measurement is commonly used to make clinical decisions with regard to glycaemic control, as HbAlc tests have not been done or the results are not yet available or are out of date. ${ }^{[13]}$ In addition, the DiabCare Africa study found that $<50 \%$ of patients with DM had had an HbAlc test as part of their management during the previous year. ${ }^{[12]}$ To this end, the availability of a rapid and accurate method of $\mathrm{HbAlc}$ evaluation is of paramount importance. ${ }^{[14]}$ According to Gaziano et al., ${ }^{[15]}$ since the late 1990s, deaths due to non-communicable diseases have increased steadily in adults aged $\geq 50$ years. A number of risk factors contributing to mortality, such as hypertension, increased 


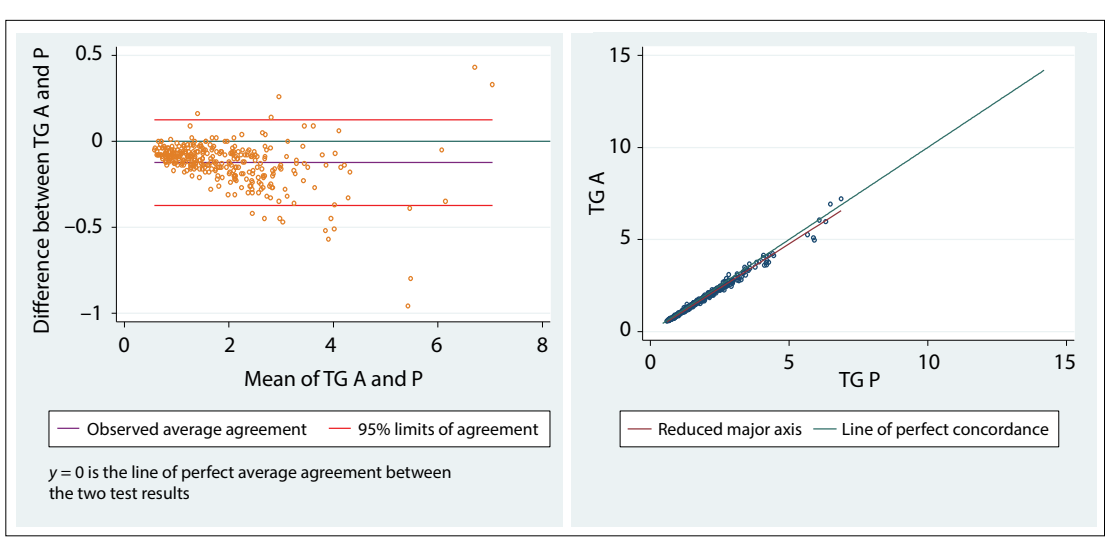

Fig. 5. Scatterplot and regression line of TG values $(\mathrm{mmol} / \mathrm{L})$ produced by the Afinion $(A)$ and Pentra $(P)$ analysers. The Bland-Altman study and linear regression analysis showed an almost perfect agreement between the two analysers. (TG = triglycerides.)

smoking prevalence, dietary changes and obesity, have been identified. These risk factors have also led to increases in symptomatic cardiovascular conditions such as stroke, ischaemic heart disease and DM, to which health policy makers have yet to provide an effective response. The global diagnostics industry is growing rapidly, since new diagnostic platforms have advanced modern healthcare in terms of the accuracy (sensitivity, specificity and reproducibility), speed and scope of diagnoses. ${ }^{[16]}$

The present study revealed a good correlation between the Afinion and Pentra analysers for the measurement of $\mathrm{HbAlc}$ levels. Our findings are supported by other published studies. A study by LentersWestra et al. ${ }^{[17]}$ evaluated seven point-of-care analysers for HbA1c (DCA Vantage, Afinion, Innova Star, Quo-Lab, Quo-Test, Cobas B101, $B$-analyst). The Afinion passed the National Glycohemoglobin Standardization Program (NGSP) criteria with two different lot numbers. In addition, the Afinion remained unaffected by the common haemoglobin variants. The study concluded that the Afinion met the performance criteria for HbAlc. ${ }^{[12]}$ Previous studies on the diagnostic accuracy of the Afinion have been conducted on paediatric samples, as described by Wood et al. ${ }^{[18]}$ In this study, the Afinion's accuracy and precision was compared with highperformance liquid chromatography (HPLC) and DCA methods. The findings showed that the Afinion generated higher $\mathrm{HbAlc}$ results when compared with HPLC, while the DCA produced lower values. At high $\mathrm{HbAlc}$ levels, the DCA tended to read lower than HPLC, but the Afinion's accuracy did not vary according to HbAlc. ${ }^{[18]}$ Jain et al.$^{[5]}$ evaluated the performance of the Afinion in a DM and CVD screening programme. In this study, the Afinion and the reference laboratory method identified similar numbers of new patients with suspected DM. In the present study, the researchers also found that the Afinion detected $\mathrm{HbAlc}$ levels that were above target levels. Similar findings were reported by Jain et al. ${ }^{[5]}$ with the Afinion being shown to overestimate HbAlc levels for certain samples. However, similar to the present study, the overestimation did not affect the overall diagnostic performance of the instrument. The Afinion is still recommended as a useful screening instrument for $\mathrm{DM}$ in a community setting, but diagnosis needs to be confirmed by an NGSP-certified method. ${ }^{[5]}$

In SA, since the prevalence of familial hypercholesterolaemia is as high as 1 in 100 in some communities, ideally everyone should undergo a full lipogram, or at minimum TC/LDL-C measurement, at least once in young adulthood (from 20 years of age). ${ }^{[3]}$ Although the measurement of lipids in the blood is a widely accepted biochemical marker for cardiovascular risk assessment and management in primary healthcare, ${ }^{[19,20]}$ testing performed in hospital laboratories may result in excess travel for the patient, sample loss and repeat clinic visits. ${ }^{[19]}$ A survey of the literature showed a limited number of studies that have investigated the performance of the Afinion analyser in measuring lipid levels. Our study is one of two suggesting that the Afinion is an acceptable POC tool for the measurement of lipids. Our findings support those of Jain et al., ${ }^{[5]}$ who compared the Afinion with the Cholestech LDX for the measurement of TC, HDL-C and triglyceride levels. The Afinion was shown to meet the performance criteria recommended by the National Education Cholestrol Program in In the present study, the readings obtained for HDL-C levels with the Afinion were shown the UK for TC and HDL-C measurements. ${ }^{[5]}$ to be slightly overestimated compared with the Pentra. Similarly, the Afinion was shown to overestimate HDL-C levels in the study by Jain et al..$^{[5]}$ Despite the overestimated values in the present study, overall the correlation between the two instruments used showed an excellent level of agreement. Our study adds to the growing body of evidence regarding the diagnostic performance of the Afinion for the measurement of lipid levels.

\section{Study limitations}

A limitation of this study is that the testing was performed in a laboratory setting by trained laboratory personnel and is not truly representative of a primary healthcare clinic setting. Field studies at primary healthcare facilities using healthcare workers (nurses) to perform the testing could be a future research direction. The study was also limited to a targeted population (older individuals). We have provided a strong rationale of why we chose to work with this population. However, future studies may need to be conducted in a more general population group. Cost-effectiveness studies on the use of the Afinion in resource-poor settings are needed.

\section{Conclusion}

The study is unique in that it provides the first report on the diagnostic performance of the Afinion AS100 analyser in measuring $\mathrm{HbAlc}$ and lipid levels in a population of HIV-infected and uninfected adults aged $\geq 50$ years in KwaZulu-Natal Province, SA. In our study population there were no significant differences in $\mathrm{HbAlc}$ and lipid levels according to HIV status. In addition, there have been no reports demonstrating the role of HIV infection and antiretroviral use in affecting the diagnostic performance of point-of-care instruments for $\mathrm{HbAlc}$ and lipid levels. This study supports the use of the Afinion as a POC test for the measurement of $\mathrm{HbAlc}$, triglycerides and HDL-C in an SA setting.

Acknowledgements. We thank all the men and women who participated in the SHIOP study, and the SHIOP study teams at the SAMRC HIV Prevention Research Unit in Durban.

Author contributions. NSA and MN developed the concept, NSA, MN and TR performed the data interpretations, TR completed the statistical analysis, and NSA wrote the article with input from MN, TR and GR.

Funding. This study was supported by the SAMRC HIV Prevention Research Unit.

Conflicts of interest. None. 
1. Yusuf S, Rangarajan S, Teo K, et al. Cardiovascular risk and events in 17 low-, middle-, and highincome countries. N Engl J Med 2014;371(9):818-827. https://doi.org/10.1056/NEJMoa1311890

2. Steyn K, Sliwa K, Hawken S, et al. Risk factors associated with myocardial infarction in Africa: Steyn K, Sliwa K, Hawken S, et al. Risk factors associated with myocardial infarction in Africa:
The INTERHEART Africa study. Circulation 2005;112(23):3554-3561. https://doi.org/10.1161/ CIRCULATIONAHA.105.563452 3. Klug EQ, Raal F, Marais A, et al. South African Dyslipidaemia Guideline Consensus Statement: A joint statement from the South African Heart Association (SA Heart) and the Lipid and Atherosclerosis Society of Southern Africa (LASSA). S Afr Fam Pract 2013;55(1):9-18. https://doi.org/10.1080/2078
6204.2013.10874296

4. Khunti K, Stone MA, Burden AC, et al. Randomised controlled trial of near-patient testing for glycated haemoglobin in people with type 2 diabetes mellitus. Br J Gen Pract 2006;56(528):511-517.

5. Jain A, Rao N, Sharifi M, et al. Evaluation of the point of care Afinion AS100 analyser in a community setting. Ann Clin Biochem 2016;54(3):331-341. https://doi.org/10.1177/0004563216661737

6. Schwartz KL, Monsur JC, Bartoces MG, West PA, Neale AV. Correlation of same-visit HbAlc tes with laboratory-based measurements: A MetroNet study. BMC Fam Pract 2005;6(1):28. https://doi. org/10.1186/1471-2296-6-28

7. Bubner TK, Laurence CO, Gialamas A, et al. Effectiveness of point-of-care testing for therapeutic control of chronic conditions: Results from the PoCT in General Practice Trial. Med J Aus 2009;190(11):624-626. https://doi.org/10.1186/1471-2296-6-28

8. Plüddemann A, Thompson M, Price CP, Wolstenholme J, Heneghan C. Point-of-care testing for the analysis of lipid panels: Primary care diagnostic technology update. Br J Gen Prac 2012;62(596):e224-e226. https://doi.org/10.3399/bjgp12X630241

9. Simbayi L, Shisana O, Rehle T, et al. South African National HIV Prevalence, Incidence and Behaviour Survey, 2012. Pretoria: Human Sciences Research Council, 2014.

10. Friedewald WT, Levy RI, Fredrickson DS. Estimation of the concentration of low-density lipoprotein cholesterol in plasma, without use of the preparative ultracentrifuge. Clin Chem 1972;18(6):499-502. 1. Johnson R, McNutt P, MacMahon S, Robson R. Use of the Friedewald formula to estimate LDLcholesterol in patients with chronic renal failure on dialysis. Clin Chem 1997;43(11):2183-2184.

12. Hird TR, Pirie FJ, Esterhuizen TM, et al. Burden of diabetes and first evidence for the utility of HbAlc for diagnosis and detection of diabetes in urban black South Africans: The Durban Diabetes Study. PLoS One 2016;11(8):e0161966. https:///doi.org/10.1371/journal.pone.0161966
13. Mash R, Ugoagwu A, Vos C, Rensburg M, Erasmus R. Evaluating point-of-care testing for glycosylated haemoglobin in public sector primary care facilities in the Western Cape, South Africa. S Afr Med I 2016;106(12):1236-1240. https://doi.org/10.7196/SAMJ.2016.v106.i12.10728

14. Motta LA, Shephard MDS, Brink J, Lawson S, Rheeder P. Point-of-care testing improves diabetes management in a primary care clinic in South Africa. Prim Care Diabetes 2017;11(3):248-253. https:// doi.org/10.1016/j.pcd.2016.09.008

15. Gaziano TA, Abrahams-Gessel S, Gomez-Olive F, et al. Cardiometabolic risk in a population of older adults with multiple co-morbidities in rural South Africa: The HAALSI (Health and Aging in Africa: Longitudinal studies of INDEPTH communities) study. BMC Public Health 2017;17(1):206. https:// doi.org/10.1186/s12889-017-4117-y

16. Thompson M, Weigl B, Fitzpatrick A, Ide N. More than just accuracy: A novel method to incorporate multiple test attributes in evaluating diagnostic tests including point of care tests. IEEE J Transl Eng Health Med 2016;4:1-8. https://doi.org/10.1109/JTEHM.2016.2570222

17. Lenters-Westra E, Slingerland RJ. Three of 7 hemoglobin Alc point-of-care instruments do not meet generally accepted analytical performance criteria. Clin Chem 2014;60(8):1062-1072. https://doi. org/10.1373/clinchem.2014.224311

18. Wood JR, Kaminski BM, Kollman C, et al. Accuracy and precision of the Axis-Shield Afinion hemoglobin Alc measurement device. J Diabetes Sci Technol 2012;6(2):380-386. https://doi. $\mathrm{rg} / 10.1177 / 193229681200600224$

19. Carey M, Markham C, Gaffney P, Boran G, Maher V. Validation of a point of care lipid analyser using a hospital based reference laboratory. Ir J Med Sci 2006;175:30. https://doi.org/10.1007/BF03167964

20. Shephard MD. Comparative performance of two point-of-care analysers for lipid testing. Clin Lab 2007;53(9-12):561-566.

Accepted 3 August 2017 